\title{
Evaluation of Midkine Expression in Dentigerous Cysts, Odontogenic Keratocysts and Different Types of Ameloblastoma
}

\author{
Naghmeh JEIROODI ${ }^{1}$, Mahshid BAGHERPOUR ${ }^{1}$, Razieh ZARE' ${ }^{1}$, Shima TORABI ARDAKANI', \\ Azadeh ANDISHEH TADBIR ${ }^{2}$
}

Department of 'Oral and Maxillofacial Pathology and '2Oral and Dental Disease, School of Dentistry, Shiraz University of Medical Sciences, SHIRAZ, IRAN

\begin{abstract}
Objective: Midkine is a heparin-binding growth factor whose expression is increased in most tumors, namely ameloblastomas. This study aimed to compare Midkine expression in different odontogenic lesions.

Material and Method: This analytical cross-sectional study was performed on 52 definitely diagnosed odontogenic lesions including 15 dentigerous cysts, 13 odontogenic keratocysts, and 17 unicystic and 5 multicystic ameloblastomas archived from 1997 to 2015. Midkine expression was examined in tissue samples through immunohistochemistry. The nonparametric Kruskal-Wallis and Mann-Whitney tests were run as appropriate $(\mathrm{P}<0.05)$.

Results: The frequency of Midkine expression was < 20\% in 7.7\%, 20-50\% in 25\%, and $>50 \%$ in $67.3 \%$ of the samples, indicating significant differences among the groups $(\mathrm{P}=0.002)$. Moreover, the expression intensity was strong in $63.5 \%$, moderate in $23.1 \%$, and weak in $13.5 \%$ of odontogenic lesion samples $(\mathrm{P}=0.071)$. The total staining score was weak in $3.8 \%$, moderate in $48.1 \%$, and strong in $48.1 \%$ of the cells, displaying significant differences between the study groups in this regard $(\mathrm{P}=0.043)$.

Conclusion: Midkine can be considered as both a differentiating factor and a molecular-targeted therapy in odontogenic lesions. Yet, further studies are required to approve the role of this cytokine in different biological and pathological stages of the tumors.
\end{abstract}

Key Words: Dentigerous Cysts, Odontogenic Keratocyst, Ameloblastoma, Unicystic, Multicystic

\section{INTRODUCTION}

Odontogenic cysts are important pathologic lesions of the oral cavity. Among the most prevalent developmental odontogenic jaw cysts are dentigerous cysts (follicular). These occur as a result of fluid accumulation between the tooth crown and reduced enamel epithelium. It is treated through enucleation and extraction of the involved tooth. The prognosis of a dentigerous cyst is excellent and rarely does it recur.

An odontogenic keratocyst is a relatively common developmental odontogenic jaw cyst originating from the remnants of dental lamina. Due to the genetic features, tendency to recur, and high aggressiveness according to WHO classification, the parakeratinized variant of odontogenic keratocysts is classified as a benign odontogenic tumor (1-3).

Ameloblastoma is a generally benign odontogenic tumor of the jaw bone. Yet, it is quite likely to relapse after extensive surgeries. Ameloblastoma is more prevalent in the

(Turk Patoloji Derg 2018, 34:158-164)

Received : 15.04.2017 Accepted : 23.11.2017 mandible than the maxilla; and rarely does it occur in an extraosseous location. Malignant ameloblastoma is quite uncommon. The etiology is still unknown. Its occurrence rate is estimated to be 0.5 in one million in a year; however, this number is estimated to be much higher in some regions such as South Africa (4-6). Most patients are 30-60 years old when diagnosed. There is no difference between the sexes concerning the distribution of the disease. Bony septa may result in a honeycomb appearance (7). Clinically and radiographically, ameloblastomas are divided into the three clinical groups of unicystic, peripheral, and solid/ multicystic, the last of which is the most frequent (8).

The multifunctional cytokine, Midkine, is a heparinbinding growth factor with low molecular weight (13 kilodalton). It contributes to formation of different organs through mesenchymal-epithelial interactions (9-12). Although the role and biological effective mechanisms of Midkine are not crystal clear, its angiogenic role in tumorigenesis has been well proven (12).

Correspondence: Azade ANDISHE TADBIR

Shiraz University of Medical Sciences, School of Dentistry,

Department of Oral and Dental Disease, SHIRAZ, IRAN

E-mail: jeiroodi@yahoo.com Phone: +989177031378 
Various investigations on Midkine expression in tumors and cancers have reported up-regulation of the gene of this cytokine in different tumors, namely Wilms tumor (13), breast cancer (14), ovarian cancer (15), gastrointestinal cancer (16), and bladder cancer (17). The findings indicate that histochemical evaluation of Midkine can be used to assess the state of cancers. This cytokine is therefore becoming one of the markers of cancer (18). So far, no research has evaluated the expression level of this marker in different odontogenic lesions with various biological behavior. Hence, the current study was designed to compare the expression of Midkine in different odontogenic lesions.

\section{MATERIALS and METHODS}

This retrospective cross-sectional study reviewed 52 paraffin-embedded tissue blocks of oral odontogenic lesions collected from the archive of the Department of Pathology, Shiraz School of Dentistry, in the time period from 1997 to 2015. The samples included 15 dentigerous cysts, 13 odontogenic keratocysts, and 17 unicystic and 5 multicystic ameloblastomas.

Having checked the hematoxylin-eosin slides, those samples with a definite diagnosis were selected. The severely inflamed cases of odontogenic keratocysts and dentigerous cysts were excluded from the study.

Immunohistochemical staining was carried out by using the Envision Labeled Peroxides System (Dako; Carpentaria, CA, USA). All samples were fixed in $10 \%$ buffered formalin. They were then hydrated in graded alcohol and rinsed with distilled water. Antigen retrieval was performed by using the Dakocytomation target retrieval solution $(\mathrm{pH}=9)$ for 9 minutes.

The endogenous peroxidase activity was inhibited by $3 \%$ $\mathrm{H} 2 \mathrm{O} 2$. Tissue sections were incubated with anti-Midkine antibody (Abcam, ab66596) at $1 / 100$ for 30 minutes. Omission of the primary antibody and hepatocellular carcinoma were respectively employed as negative and positive control.

An oral and maxillofacial pathologist examined the slides randomly in the epithelium by using an optical microscope at 100 and 400X. The cells were counted in a fixed single blind fashion in 5 microscopic fields. The antibody reactive cells were then counted in each field. Finally, the mean percentage of stained cells as well as the mean staining intensity was calculated.

The qualitative scores of immunohistochemical staining were quantitatively analyzed by using the SID score (staining-intensity-distribution); i.e., Distribution + Staining Intensity of the stained cells. The staining of cells in all samples included the cell cytoplasm and membrane. Only those cells with obvious staining were considered as stained positive.

To determine the frequency of stained cells, the lack of staining was scored as 0 , staining $<20 \%$ scored as $1+, 25$ $50 \%$ as $2+$, and $>50 \%$ was scored as $3+$. To determine the staining intensity, lack of staining was scored as 0 , weak staining as $1+$, moderate as $2+$, and strong staining was scored as $3+$. To calculate the total lesion score, the summation of staining and intensity $<3$ was considered to be weak, 3-5 was moderate, and $=6$ was strong.

The distribution of data was evaluated through descriptive statistics. Having assessed the abnormality of data, the Kruskal-Wallis nonparametric test was used to compare the staining ability, staining intensity, and total scores. If needed, Mann-Whitney multiple comparison tests were run to compare the marker expression among the four groups. The obtained data was analyzed by using the SPSS software, version $18(\mathrm{P}<0.05)$.

\section{RESULTS}

A total of 52 cases of odontogenic lesions were taken from the archive of the Department of Pathology of Shiraz School of Dentistry. The suspicious and deficient cases were excluded. The frequency and distribution of cases with definite diagnosis was in four groups of dentigerous cyst, odontogenic keratocyst, unicystic and multicystic ameloblastoma. The patients' demographic data is displayed in Table I.

Age was described using mean \pm SD and sex was described using frequency (\%). There were no significant differences for age and sex between the groups (Table II).

The results of this study revealed that the Midkine factor is highly expressed in odontogenic lesions. The frequency of staining can be seen in Table III. Significant differences were observed in the staining percentage (Midkine expression) among the study groups $(\mathrm{p}=0.002)$. The Mann-Whitney test showed higher expression of Midkine in multicystic ameloblastoma than dentigerous cyst $(\mathrm{p}=0.009)$. Also the Mann-Whitney test showed higher expression of Midkine in OKC in comparison with DC $(p=0.01)$. Midkine expression in multicystic lesions was higher than unicystic lesions $(\mathrm{p}=0.033)$. Our data showed higher expression of OKC compared with unicystic lesions $(\mathrm{p}=0.005)$.

The pathologic evaluations revealed that the multicystic ameloblastoma included follicular and plexiform samples. All samples in this groups showed positive staining. Immunoreactivity was mostly observed in ameloblast-like and stellate-reticulum like cells. 
The staining intensity of Midkine factor is shown in Table III. However, Based on Mann-Whitney test, the difference was not significant among the groups $(\mathrm{P}=0.071)$

The staining score can be seen in Table III. The KruskalWallis test revealed significant differences between the staining scores of the four types of odontogenic lesions $(\mathrm{P}=0.043)$. Comparision between multicystic ameloblastoma and dentigerous cyst showed higher expression of Midkine in multicystic ameloblastoma $(\mathrm{p}=0.010)$. Also higher Midkine expression was seen in OKC than in dentigerous cyst $(\mathrm{p}=0.048)$.

Table I: The demographic data of the patients

\begin{tabular}{lcccc}
\hline & Number & Mean Age & Male & Female \\
\hline Unicystic Ameloblastoma & $17(32.7)$ & $29.94 \pm 9.32$ & $6(37.5)$ & $10(62.5)$ \\
\hline Multicystic Ameloblastoma & $7(13.5)$ & $36.80 \pm 21.18$ & $2(33.3)$ & $4(66.7)$ \\
\hline Odontogenic Keratocyst & $13(25.00)$ & $34.15 \pm 12.09$ & $5(38.5)$ & $8(61.5)$ \\
\hline Dentigerous Cyst & $15(28.8)$ & $30.13 \pm 10.72$ & $9(60.00)$ & $6(40.00)$ \\
\hline Total & $52(100.00)$ & $31.78 \pm 11.82$ & $22(44.00)$ & $28(56.00)$ \\
\hline
\end{tabular}

Data are presented as mean \pm SD or number (\%)

Table II: Comparison of age and sex variables between different tumor types

\begin{tabular}{llccccc}
\hline & & Unicystic & Multicystic & OKC & Dentigerous cyst & P Value \\
\hline \multirow{2}{*}{ Age } & & $29.94 \pm 9.32$ & $36.80 \pm 21.18$ & $34.15 \pm 12.09$ & $30.13 \pm 10.72$ & 0.559 \\
\cline { 1 - 6 } Sex & F & $10(62.5 \%)$ & $4(66.7 \%)$ & $8(61.5 \%)$ & $6(40.0 \%)$ & \multirow{2}{*}{0.518} \\
\cline { 2 - 6 } & M & $6(37.5 \%)$ & $3(33.3 \%)$ & $5(38.5 \%)$ & $9(60.0 \%)$ & \\
\hline
\end{tabular}

Age was described using mean \pm SD and sex was described using frequency (\%).

Table III: Comparison of staining percentage, staining density and total scores of the studied odontogenic cysts

\begin{tabular}{|c|c|c|c|c|c|c|}
\hline & $\begin{array}{c}\text { Unicystic } \\
\text { Ameloblastoma } \\
\text { n (\%) } \\
\end{array}$ & $\begin{array}{c}\text { Multicystic } \\
\text { Ameloblastoman } \\
(\%)\end{array}$ & $\begin{array}{c}\text { Odontogenic } \\
\text { Keratocyst } \\
\text { n (\%) } \\
\end{array}$ & $\begin{array}{c}\text { Dentigerous } \\
\text { Cyst } \\
\text { n (\%) } \\
\end{array}$ & $\begin{array}{l}\text { Total } \\
\text { n (\%) }\end{array}$ & $\begin{array}{c}\mathbf{P} \\
\text { Value }\end{array}$ \\
\hline \multicolumn{7}{|l|}{ Percentage } \\
\hline n $<20 \%$ & $4(23.5)$ & $0(0.00)$ & $0(0.00)$ & $0(0.00)$ & $4(7.70)$ & \\
\hline $20 \%<x<50 \%$ & $4(23.5)$ & $0(0.00)$ & $0(0.00)$ & $9(60.00)$ & $13(25.01)$ & \\
\hline$n>50 \%$ & $9(52.9)$ & $7(100.00)$ & $13(100.00)$ & $6(40.00)$ & $35(67.30)$ & \\
\hline Total & $17(100.00)$ & $7(100.00)$ & $13(100.00)$ & $15(100.00)$ & $52(100.00)$ & \\
\hline Rank (Median:min-max) & $21 / 71(3: 1-3)$ & $35(3: 3-3)$ & $35(3: 3-3)$ & $20 / 60(2: 2-3)$ & & 0.002 \\
\hline \multicolumn{7}{|l|}{ Intensity } \\
\hline Weak & $2(11.8)$ & $0(0.00)$ & $0(0.00)$ & $5(33.3)$ & $7(13.5)$ & \\
\hline Moderate & $1(5.9)$ & $1(14.3)$ & $7(53.8)$ & $3(20.00)$ & $12(23.1)$ & \\
\hline Strong & $14(82.4)$ & $6(85.7)$ & $6(46.2)$ & $7(46.7)$ & $33(63.5)$ & \\
\hline Total & $17(100.0)$ & $7(100.0)$ & $13(100.0)$ & $15(100.0)$ & $52(100.0)$ & \\
\hline Rank (Median:min-max) & $30 / 91(3: 1-3)$ & $32 / 79(3: 2-3)$ & $23 / 88(2: 2-3)$ & $20 / 83(2: 1-3)$ & & 0.071 \\
\hline \multicolumn{7}{|l|}{ Score } \\
\hline 2 & $2(11.8)$ & $0(0)$ & $0(0)$ & $0(0)$ & $2(3.8)$ & \\
\hline 3 & $0(0)$ & $0(0)$ & $0(0)$ & $5(33.3)$ & $5(9.6)$ & \\
\hline 4 & $3(17.6)$ & $0(0)$ & $0(0)$ & $1(6.7)$ & $4(7.7)$ & \\
\hline 5 & $3(17.6)$ & $1(14.3)$ & $7(53.8)$ & $5(33.3)$ & $16(30.8)$ & \\
\hline 6 & $9(52.9)$ & $6(85.7)$ & $6(46.2)$ & $4(26.7)$ & $25(48.1)$ & \\
\hline Total & $17(100.0)$ & $7(100.0)$ & $13(100.0)$ & $15(100.0)$ & $52(100.0)$ & \\
\hline Rank (Median:min-max) & $26 / 47(6: 2-6)$ & $37 / 07(6: 5-6)$ & $28 / 96(5: 5-6)$ & $19 / 47(5: 3-6)$ & & 0.043 \\
\hline
\end{tabular}


The unicystic form of ameloblastoma contained mural, luminal, and intraluminal parts. The staining was positive in the epithelial lining. The staining was detected to be higher than $50 \%$ in all 13 cases of odontogenic keratocysts. The cells showed staining in the cytoplasm of the basal and parabasal cells. In dentigerous cysts, 6 out of 15 cases showed $>50 \%$ staining (Figure $1 \mathrm{~A}-\mathrm{D}$ ).

\section{DISCUSSION}

Midkine is a heparin-binding cytokine that enhances growth, survival, migration and other activities of the target cells. The present research reviewed the expression level of the Midkine gene in oral odontogenic lesions including dentigerous cyst, odontogenic keratocyst, unicystic and multicystic ameloblastomas. The obtained results revealed that Midkine factor is highly expressed in odontogenic lesions.
Few studies have investigated the expression of the Midkine cytokine in odontogenic lesions such as ameloblastoma. Gramage et al. observed the regulation of expression of the Midkine gene and protein through epithelial-mesenchymal interaction in the tooth germ and suggested that Midkine plays an imperative role in the molecular cascade that controls the growth and development of the tooth (19).

Scheper et al. studied the Midkine expression in 34 patients with ameloblastoma and 4 cases of ameloblastic carcinoma. They found that the mentioned marker was expressed in $67 \%$ of cases, out of which $23.5 \%$ was weak, $14.7 \%$ was moderate, and $29.4 \%$ was strong. A statistically significant difference was observed between unicystic and multicystic cases (18).The present study was different in assessing all types of odontogenic lesions, and achieving total score of strong staining in $48.1 \%$ of samples.
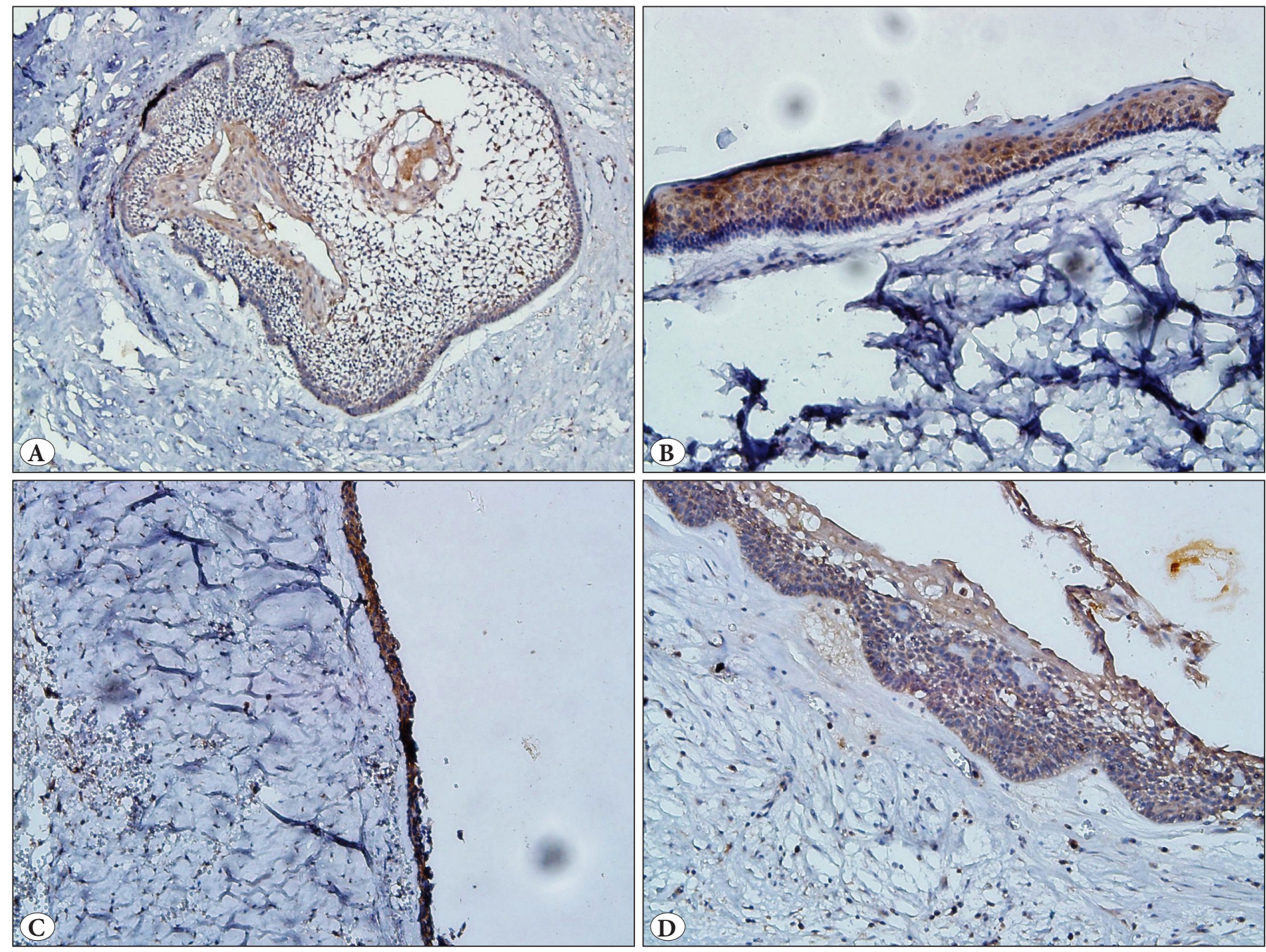

Figure 1: Cytoplasmic Midkine Staining in Odontogenic Lesions Tissue Samples. A) Ameloblastoma (IHC; x100). B) Odontogenic keratocyst (IHC; x200). C) Dentigerous cyst (IHC; x100). D) Unicystic ameloblastoma (IHC; x200) 
In the current study, expression $>50 \%$ was observed in $52.9 \%$ of unicystic ameloblastomas, $100 \%$ of both multicystic ameloblastomas and odontogenic keratocyst tumors, and $40 \%$ of dentigerous cysts. Overall, $67.3 \%$ of odontogenic cysts showed above 50\% Midkine expression. The difference was significant among the study groups.

Sandra et al. investigated the expression and performance of Midkine in 37 cases of tissue ameloblastoma, and ameloblastoma cell lines (AM-1), and altered HPV-16DNA cell lines. They found through the immunohistochemical method that Midkine is expressed in the outer layers of ameloblastoma-like and AM-1 cells in 70\% of cases (20).

Likewise, Fujita et al. examined Midkine expression in different odontogenic lesions including 50 ameloblastoma samples. Midkine was located in the columnar cell cytoplasm in $54.5 \%$ of cases (30 out of 55) (21). Our results are therefore consistent with the findings of other studies concerning the changes in Midkine expression in odontogenic tumors.

An important unappreciated hint in recent studies is the relationship between the Midkine cytokine expression and the pathological and clinical parameters. We investigated in the present study whether this protein contributes to different pathologic behavior. It was revealed that Midkine expression was stronger in multi-cystic lesions and odontogenic keratocysts than in dentigerous cysts. The finding suggests that this protein is expressed at a higher rate in the presence of invasive behavior.

There are studies on the exact route of signal transmission such as Notch that might play a role in creation of different phenotypes of odontogenic lesions. However, among the limitations of the current study was the inaccessibility of further data; hence, we could not elaborate on the relation between Midkine expression and the severity of tumor invasion.

Fujita et al. reported that Midkine was expressed in keratinized cells of acanthomatous ameloblastoma and keratocyst odontogenic tumors. In mixed odontogenic tumors, except for odontoma, the immunoreactions to Midkine were noted in the epithelial follicles surrounding the ectomesenchymal odontogenic tissue (21). Accordingly, Midkine can contribute to the growth and activities of odontogenic tumors, as well as to cell differentiation through molecular mechanisms similar to tooth morphogenesis.

The differentiation value of Midkine in odontogenic lesions is among the disputable issues. In the present study, Midkine was expressed in both the cytoplasm and membrane. No difference was mentioned between the two different Midkine expression patterns (cytoplasmic and membranous). Hence, further studies are required to clarify the significance of various Midkine expression patterns.

The presence of similar inflammatory and epithelial cells might interfere with the differential diagnosis of these lesions. However, pairwise comparison of the staining percentage of Midkine cytokine in these lesions revealed significant differences between unicystic and multicystic ameloblastoma $(\mathrm{P}=0.033)$ and odontogenic keratocyst ( $\mathrm{P}=0.001)$.

Significant differences were also observed in the expression level of Midkine between odontogenic keratocyst and dentigerous cyst $(\mathrm{P}=0.001)$, as well as multi-cystic ameloblastoma and dentigerous cyst $(\mathrm{P}=0.009)$. Such significant differences can imply that Midkine can be used as a differential factor in these lesions.

Other studies reported that this cytokine was expressed in diseased tissues by inflammatory cells like macrophages, lymphocytes, and endothelial cells whereas such an expression did not occur in normal tissues (22). Increase and changes in Midkine expression were also reported in other diseases and cancers, namely esophageal squamous cancer $(14,17,18,23)$, squamous cell carcinoma (24), and meningioma (25). This proves its role as a candidate gene in targeted therapy.

The results of studies suggest that immunohistochemical evaluation of Midkine can be used as a way to examine the cancer status; this cytokine is therefore becoming a marker of cancer. Accordingly, Midkine can be used as a potential target therapy in odontogenic lesions where therapeutic measures can be taken according to its increase or decrease.

Scheper et al. found that Midkine expression increased in human ameloblastoma (18). Meanwhile, Hatori et al. reported that the expression of this cytokine increased in inflammatory dental granuloma (22). They also added that propagation of this cytokine was required to prevent apoptosis. Hence, reduced expression of this cytokine by genotoxic drugs increased the number of cancerous cells that were entering the apoptosis stage. Seemingly, the synergism effect of some medications can help treat the cancer through targeting the DNA and Midkine gene in humans.

Previous studies have revealed that genetic changes in the path of the AKT protein resulted in hyperactivity of this protein, as already reported in a number of cancers. In the 
laboratory environment, Midkine can phosphorylase and activate the AKT kinase protein and increase the cell growth through the SRC and extracellular path. Accordingly, the cellular growth and proliferation continues and apoptosis is reduced. As declared in a previous study, stimulation of Midkine in cultured meningioma cells led to phosphorylation of AKT.

Midkine can also protect the meningioma cells against apoptotic death by decreasing active caspase-3. Therefore, targeting the Midkine in the signal transmission route and blocking it can be a useful therapeutic approach in odontogenic lesions, particularly those with significant invasion.

In conclusion, the results of this study showed that Midkine is expressed in several odontogenic lesions; particularly in more invasive ones, this protein can indicate the progress and survival of tumor behavior. Midkine can be used as a differential method between odontogenic lesions when hematoxylin-eosin staining does not work for differentiating the lesions. Moreover, Midkine can be a targeted-molecular therapy for odontogenic lesions. Yet, further studies are required to approve the role of this gene in different biological and pathological stages of tumor.

\section{ACKNOWLEDGMENTS}

The authors thank the Vice-Chancellery of Shiraz University of Medical Science for supporting this research (Grant\# 8895166). This manuscript is based on the thesis of Mahshid Bagherpour for partial fulfillment of DDS degree. The authors are grateful to Dr. M. Vossoughi from the Dental Research Development Center of the Dental School for the statistical analysis and Ms. Farzaneh Rasouli for her help with the English editing of the manuscript.

\section{CONFLICT of INTEREST}

The authors declare no conflict of interest.

\section{REFERENCES}

1. Cheng YS, Rees T, Wright J. A review of research on salivary biomarkers for oral cancer detection. Clin Transl Med. 2014;3:3.

2. Gaitan-Cepeda LA, Quezada-Rivera D, Tenorio-Rocha F, LeyvaHuerta ER. Reclassification of odontogenic keratocyst as tumour. Impact on the odontogenic tumours prevalence. Oral Dis. 2010;16:185-7.

3. Sasaki R, Miyashita T, Matsumoto N, Fujii K, Saito K, Ando T. Multiple keratocystic odontogenic tumors associated with nevoid basal cell carcinoma syndrome having distinct PTCH1 mutations: A case report. Oral Surg Oral Med Oral Pathol Oral Radiol Endod. 2010;110:e41-6.
4. Hidalgo-Sanchez O, Leco-Berrocal MI, Martinez-Gonzalez JM. Metaanalysis of the epidemiology and clinical manifestations of odontomas. Med Oral Patol Oral Cir Bucal. 2008;13:E730-4.

5. Lascane NA, Sedassari BT, Alves Fde A, Gallottini MH, de Sousa SC. Peripheral ameloblastoma with dystrophic calcification: An unusual feature in non-calcifying odontogenic tumors. Braz Dent J. 2014;25:253-6.

6. Gunawardhana KS, Jayasooriya PR, Tilakaratne WM. Diagnostic dilemma of unicystic ameloblastoma: Novel parameters to differentiate unicystic ameloblastoma from common odontogenic cysts. J Investig Clin Dent. 2014;5:220-5.

7. Seethala RR, Stenman G. Update from the 4th Edition of the World Health Organization Classification of Head and Neck Tumours: Tumors of the Salivary Gland. Head Neck Pathol. 2017;11:55-67.

8. Almeida Rde A, Andrade ES, Barbalho JC, Vajgel A, Vasconcelos BC. Recurrence rate following treatment for primary multicystic ameloblastoma: Systematic review and meta-analysis. Int J Oral Maxillofac Surg. 2016;45:359-67.

9. Kadomatsu K, Muramatsu T. Midkine and pleiotrophin in neural development and cancer. Cancer Lett. 2004;204:127-43.

10. Wu GC, Yuan H, Pan HF, Ye DQ. Elevated plasma midkine and pleiotrophin levels in patients with systemic lupus erythematosus. Oncotarget. 2016.

11. Haynes L, Rumsby M. The pleiotropin/midkine family of cytokines: Role in glial-neuronal signalling. Prog Brain Res. 2001;132:313-24.

12. Li F, Tian P, Zhang J, Kou C. The clinical and prognostic significance of midkine in breast cancer patients. Tumour Biol. 2015;36:9789-94.

13. Choi YW, Kim YH, Lee J, Soh EY, Park TJ, Kim JH. Strong immunoexpression of midkine is associated with multiple lymph node metastases in BRAFV600E papillary thyroid carcinoma. Hum Pathol. 2015;46:1557-65.

14. Chen J, Li J, Li W, Hu H. Expression of midkine and microvessel density in salivary adenoid cystic carcinoma. Hua Xi Kou Qiang Yi Xue Za Zhi. 2016;34:189-93.

15. Jham BC, Costa NL, Silva JM, de Miranda AC, Oliveira JC, Silva TA, Batista AC. Midkine expression in oral squamous cell carcinoma and leukoplakia. J Oral Pathol Med. 2012;41:21-6.

16. Shao H, Yu X, Wang C, Wang Q, Guan H. Midkine expression is associated with clinicopathological features and BRAF mutation in papillary thyroid cancer. Endocrine. 2014;46:285-91.

17. Chiu TJ, Chen YJ, Rau KM, Chen CH, Chien CY, Li SH, Tsai HT, Eng HL. Midkine neurite growth-promoting factor 2 expression as a potential prognostic marker of adjuvant therapy in head and neck squamous cell carcinoma. Biomarkers. 2013;18:687-98.

18. Scheper MA, Duarte EC, Intapa C, Zhang M, Nascimento LM, Almeida TP, Gomes AC, Song S, Chaisuparat R, Batista AC, Jham BC. Expression of midkine in ameloblastomas and its correlation with clinicopathologic parameters. Oral Surg Oral Med Oral Pathol Oral Radiol. 2012;114:497-502.

19. Gramage E, Li J, Hitchcock P. The expression and function of midkine in the vertebrate retina. Br J Pharmacol. 2014;171:91323. 
20. Sandra F, Harada H, Nakamura N, Ohishi M. Midkine induced growth of ameloblastoma through MAPK and Akt pathways. Oral Oncol. 2004;40:274-80.

21. Fujita S, Seki S, Fujiwara M, Ikeda T. Midkine expression correlating with growth activity and tooth morphogenesis in odontogenic tumors. Hum Pathol. 2008;39:694-700.

22. Hatori K, Takeichi O, Ogiso B, Maeno M, Komiyama K. Midkine expression in human periapical granulomas. J Endod. 2011;37:781-5.
23. Jin Z, Lahat G, Korchin B, Nguyen T, Zhu QS, Wang X, Lazar AJ, Trent J, Pollock RE, Lev D. Midkine enhances soft-tissue sarcoma growth: A possible novel therapeutic target. Clin Cancer Res. 2008;14:5033-42.

24. Ruan M, Ji T, Wu Z, Zhou J, Zhang C. Evaluation of expression of midkine in oral squamous cell carcinoma and its correlation with tumour angiogenesis. Int J Oral Maxillofac Surg. 2007;36:159-64.

25. Tong Y, Mentlein R, Buhl R, Hugo HH, Krause J, Mehdorn HM, Held-Feindt J. Overexpression of midkine contributes to anti-apoptotic effects in human meningiomas. J Neurochem. 2007;100:1097-107. 